\title{
A New Approach for a Class of the Blasius Problem via a Transformation and Adomian's Method
}

\author{
Abdelhalim Ebaid and Nwaf Al-Armani \\ Department of Mathematics, Faculty of Science, University of Tabuk, P.O. Box 741, Tabuk 71491, Saudi Arabia \\ Correspondence should be addressed to Abdelhalim Ebaid; aebaid@ut.edu.sa
}

Received 19 April 2013; Accepted 10 July 2013

Academic Editor: Rodrigo Lopez Pouso

Copyright (C) 2013 A. Ebaid and N. Al-Armani. This is an open access article distributed under the Creative Commons Attribution License, which permits unrestricted use, distribution, and reproduction in any medium, provided the original work is properly cited.

\begin{abstract}
The main feature of the boundary layer flow problems is the inclusion of the boundary conditions at infinity. Such boundary conditions cause difficulties for any of the series methods when applied to solve such problems. To the best of the authors' knowledge, two procedures were used extensively in the past two decades to deal with the boundary conditions at infinity, either the Padé approximation or the direct numerical codes. However, an intensive work is needed to perform the calculations using the Padé technique. Regarding this point, a new idea is proposed in this paper. The idea is based on transforming the unbounded domain into a bounded one by the help of a transformation. Accordingly, the original differential equation is transformed into a singular differential equation with classical boundary conditions. The current approach is applied to solve a class of the Blasius problem and a special class of the Falkner-Skan problem via an improved version of Adomian's method (Ebaid, 2011). In addition, the numerical results obtained by using the proposed technique are compared with the other published solutions, where good agreement has been achieved. The main characteristic of the present approach is the avoidance of the Padé approximation to deal with the infinity boundary conditions.
\end{abstract}

\section{Introduction}

During the past two decades much effort has been spent in the numerical treatment of boundary value problems over an unbounded domain. In fact, these problems arise very frequently in many fields such as in fluid dynamics, aerodynamics, and quantum mechanics. A few notable examples are the Blasius and Falkner-Skan equations. The Blasius equation is one of the basic equations in fluid dynamics. It describes the velocity profile of the fluid in the boundary layer theory $[1,2]$ on a half-infinite interval. Several analytical and numerical methods have been proposed in [1-11] to handle this problem. The two forms of the Blasius problem are represented by the same differential equation with different sets of boundary conditions, as will be indicated later. The main feature of the Blasius problem is the existence of the boundary conditions at infinity. Such conditions at infinity cause difficulties for any of the series methods, such as the Adomian decomposition method [12-14] and the differential transformation method (or the Taylor series method) $[15,16]$.
This is because the infinity boundary condition cannot be imposed directly in the series, where the Padé approximation should be established before applying the boundary condition at infinity. It was observed in the past two decades that many authors [17-25] have been resorted to either the Padé technique or some numerical methods to treat the boundary conditions at infinity. Although the results obtained by using the Padé technique were accurate in many cases, a massive computational work was needed to obtain accurate approximate solutions. A possible way to avoid the Padé technique is to change the boundary conditions at infinity into classical conditions. Therefore, a suggestion is proposed in this paper to transform the domain of the problem from an unbounded domain into a bounded one with the help of a simple transformation.

According to the suggested transformation, the original Blasius equation is transformed into a system of two singular differential equations. Hence, the two mentioned forms are described by this system with two different sets of boundary conditions at classical point. The transformed singular system 
will be solved by a recent version of the ADM [26]. The first form of the original Blasius problem is given by [6]

$$
f^{\prime \prime \prime}(\eta)+\frac{1}{2} f(\eta) f^{\prime \prime}(\eta)=0
$$

subject to the following boundary conditions:

$$
f(0)=0, \quad f^{\prime}(0)=1, \quad f^{\prime}(\infty)=0,
$$

while the second form is given by (1) with the following boundary conditions:

$$
f(0)=0, \quad f^{\prime}(0)=0, \quad f^{\prime}(\infty)=1 .
$$

A class of Blasius problem is given by

$$
f^{\prime \prime \prime}(\eta)+\gamma f(\eta) f^{\prime \prime}(\eta)=0,
$$

subject to the following boundary conditions:

$$
f(0)=0, \quad f^{\prime}(0)=1-\epsilon, \quad f^{\prime}(\infty)=\epsilon,
$$

where $\gamma$ and $\epsilon$ are finite constants. This class will be studied for $\epsilon \in[0,1]$. Here, it is noted that (2) and (3) are special examples of (5) for $\epsilon=0$ and $\epsilon=1$, respectively. In addition, the class (4)-(5) reduces to the two forms of the Blasius problem when $(\gamma=1 / 2, \epsilon=0)$ and $(\gamma=1 / 2, \epsilon=1)$, respectively. At the same time, when $\gamma=1$, the suggested class reduces to a special class of the Falkner-Skan problem, at $\delta=0$, which is well known as [27]

$$
f^{\prime \prime \prime}(\eta)+f(\eta) f^{\prime \prime}(\eta)+\delta\left[\epsilon^{2}-\left(f^{\prime}(\eta)\right)^{2}\right]=0,
$$

with the class of boundary conditions (5), where $\delta$ refers to the pressure gradient parameter, while $\epsilon$ refers to the velocity ratio parameter, $\epsilon=U_{\infty} /\left(U_{\infty}+U_{w}\right)$. Equation (6) with the boundary conditions (5) is a new version of the Falkner-Skan equation relating free stream velocity $U_{\infty}$ to composite reference velocity, that is, sum of the velocities of stretching boundary $U_{w}$ and free stream $U_{\infty}$. In order to use the improved Adomian's method [26] to solve the class (4)(5), we first transform the governing equation (4) into the following system of differential equations:

$$
\begin{gathered}
f^{\prime}(\eta)=u(\eta), \\
u^{\prime \prime}(\eta)+\gamma f(\eta) u^{\prime}(\eta)=0 .
\end{gathered}
$$

Here, we may indicate that in the theory of the boundary layer, it is usually important to get information about three quantities: the skin-friction coefficient $f^{\prime \prime}(0)$, the fluid velocity $f^{\prime}(\eta)$, and the stream function $f(\eta)$. Also, it is well known that at $\epsilon=1$ the problem reduces to one of the two forms of the Blasius problem which has been studied extensively during the past decades.

\section{A Transformation and a New System}

The unbounded domain of the independent variable $\eta \in$ $[0, \infty)$ can be changed into a bounded one by using a new independent variable $t$ (say) $\in[0,1)$ using the transformation $t=1-e^{-\eta}$. Accordingly, the governing system should be expressed in terms of the new variable $t$. In order to do that, we introduce the following relations between the derivatives with respect to $\eta$ and the derivatives with respect to $t$ :

$$
\begin{gathered}
\frac{d}{d \eta}(\square)=(1-t) \frac{d}{d t}(\square), \\
\frac{d^{2}}{d \eta^{2}}(\square)=(1-t)^{2} \frac{d^{2}}{d t^{2}} \text { (口) }-(1-t) \frac{d}{d t}(\square) .
\end{gathered}
$$

The relations given by (8) are obtained by using the chain rule in the differential calculus. Therefore, the system (7) becomes

$$
\begin{gathered}
f^{\prime}(t)=\left(\frac{1}{1-t}\right) u(t), \\
u^{\prime \prime}(t)=\left(\frac{1}{1-t}\right) u^{\prime}(t)-\gamma\left(\frac{1}{1-t}\right) f(t) u^{\prime}(t),
\end{gathered}
$$

subject to the following set of boundary conditions:

$$
f(0)=0, \quad u(0)=1-\epsilon, \quad u(1)=\epsilon .
$$

Equation (9) with the initial condition $f(0)=0$ can be easily integrated as an initial value problem, while (10) with the boundary conditions given in (11) should be solved as a two-point boundary value problem. In this regard, the improved Adomian decomposition method is suggested to deal with such a singular two-point boundary value problem. Before launching into the the main idea of this paper, we give an analysis for the improved Adomian decomposition method in the next section to solving (10) with general twopoint boundary conditions $u(a)=\alpha$ and $u(b)=\beta$.

\section{The Improved Adomian Decomposition Method}

Consider the second order differential equation:

$$
u^{\prime \prime}(t)+p(t) u^{\prime}(t)+q(t) f(t) u^{\prime}(t)=0,
$$

subject to the boundary conditions

$$
u(a)=\alpha, \quad u(b)=\beta,
$$

where at least one of the functions $p(t)$ and $q(t)$ has a singular point and $f(t)$ is an unspecified function. In order to apply the approach suggested in [26], we first rewrite (12) as

$$
u^{\prime \prime}(t)=-p(t) u^{\prime}(t)-q(t) f(t) u^{\prime}(t) .
$$

Now, suppose that $p(t)$ and $q(t)$ have the same singular point ( $t=t_{0}$, say), Ebaid [26] proposed the following inverse operator to solve (14) with the boundary conditions (13):

$$
\begin{array}{r}
L^{-1}[\cdot]=\int_{a}^{t} d t^{\prime} \int_{c}^{t^{\prime}}[\cdot] d t^{\prime \prime}-\frac{t-a}{b-a} \int_{a}^{b} d t^{\prime} \int_{c}^{t^{\prime}}[\cdot] d t^{\prime \prime}, \\
a \neq b, \quad c \text { (arbitrary) } \neq t_{0} .
\end{array}
$$


Operating both sides of (14) with this inverse operator, we have

$$
\begin{aligned}
u(t) & -u(a)-\frac{t-a}{b-a}[u(b)-u(a)] \\
& =-L^{-1}\left[p(t) u^{\prime}(t)+q(t) f(t) u^{\prime}(t)\right],
\end{aligned}
$$

which can be rewritten as

$$
\begin{aligned}
u(t)= & \alpha+\frac{t-a}{b-a}(\beta-\alpha) \\
& -L^{-1}\left[p(t) u^{\prime}(t)+q(t) f(t) u^{\prime}(t)\right] .
\end{aligned}
$$

Based on Adomian's method, the solutions $u(t)$ and $f(t)$ of system (9)-(10) are assumed in the following form:

$$
u(t)=\sum_{n=0}^{\infty} u_{n}(t), \quad f(t)=\sum_{n=0}^{\infty} f_{n}(t)
$$

Inserting these series into (17), we obtain

$$
\begin{aligned}
\sum_{n=0}^{\infty} u_{n}(t)= & \alpha+\frac{t-a}{b-a}(\beta-\alpha) \\
& -L^{-1}\left[p(t) \sum_{n=0}^{\infty} u_{n}^{\prime}(t)+q(t) \sum_{n=0}^{\infty} \sum_{i=0}^{n} f_{i}(t) u_{n-i}^{\prime}(t)\right] .
\end{aligned}
$$

Substituting $p(t)=-(1 /(1-t))$ and $q(t)=\gamma(1 /(1-t))$ into the last equation yields

$$
\begin{aligned}
\sum_{n=0}^{\infty} u_{n}(t)=\alpha+\frac{t-a}{b-a}(\beta-\alpha) & \\
+L^{-1} & {\left[\left(\frac{1}{1-t}\right) \sum_{n=0}^{\infty} u_{n}^{\prime}(t)\right.} \\
& \left.\quad \gamma\left(\frac{1}{1-t}\right) \sum_{n=0}^{\infty} \sum_{i=0}^{n} f_{i}(t) u_{n-i}^{\prime}(t)\right] .
\end{aligned}
$$

To overcome the difficulty of the singular point, we may replace the function $1 /(1-t)$ with the series form $\sum_{n=0}^{\infty} t^{n}$, where $t \in[0,1)$. Thus, we have

$$
\begin{aligned}
& \sum_{n=0}^{\infty} u_{n}(t)=\alpha+\frac{t-a}{b-a}(\beta-\alpha) \\
&+L^{-1}\left[\sum_{n=0}^{\infty} \sum_{i=0}^{n} t^{n-i} u_{i}^{\prime}(t)\right. \\
&\left.\quad-\gamma \sum_{n=0}^{\infty}\left(\sum_{j=0}^{n} \sum_{i=0}^{j} t^{n-j} f_{i}(t) u_{j-i}^{\prime}(t)\right)\right] .
\end{aligned}
$$

According to the modified decomposition method [18], the solution $u(t)$ can be evaluated by using the recurrence scheme:

$$
\begin{gathered}
u_{0}(t)=\alpha, \\
u_{1}(t)=\frac{t-a}{b-a}(\beta-\alpha)+L^{-1}\left[u_{0}^{\prime}(t)-\gamma f_{0}(t) u_{0}^{\prime}(t)\right], \\
u_{n+1}(t)=L^{-1}\left[\sum_{i=0}^{n} t^{n-i} u_{i}^{\prime}(t)-\gamma\left(\sum_{j=0}^{n} \sum_{i=0}^{j} t^{n-j} f_{i}(t) u_{j-i}^{\prime}(t)\right)\right], \\
n \geq 1 .
\end{gathered}
$$

On integrating (9) with respect to $t$ form 0 to $t$, it then follows that

$$
f(t)=f(0)+\int_{0}^{t}\left(\frac{1}{1-z}\right) u(z) d z .
$$

Hence, $f(t)$ is given by the recurrence scheme:

$$
f_{0}(t)=0, \quad f_{n+1}(t)=\int_{0}^{t} z^{n-i} u_{i}(z) d z, \quad n \geq 0 .
$$

The algorithms given by (22) and (24) are applied in the next section to construct the approximate solutions.

\section{Applications}

4.1. A Class of the Blasius Problem. Here, we show how to implement (22) and (24) to solve the class of the Blasius problem. On substituting $\gamma=1 / 2, a=0, b=1, \alpha=1-\epsilon$, and $\beta=\epsilon$ into (22), and using (24) we obtain

$$
\begin{gathered}
u_{0}(t)=1-\epsilon, \\
f_{0}(t)=0, \\
u_{1}(t)=(2 \epsilon-1) t, \\
f_{1}(t)=(1-\epsilon) t, \\
u_{n+1}(t)=L^{-1}\left[\sum_{i=0}^{n} t^{n-i} u_{i}^{\prime}(t)-\frac{1}{2}\left(\sum_{j=0}^{n} \sum_{i=0}^{j} t^{n-j} f_{i}(t) u_{j-i}^{\prime}(t)\right)\right], \\
f_{n+1}(t)=\int_{0}^{t}\left(\frac{1}{1-z}\right) u_{n}(z) d z, \quad n \geq 1, \\
L^{-1}[\cdot]=\int_{0}^{t} d t^{\prime} \int_{c}^{t^{\prime}}[\cdot] d t^{\prime \prime}-t \int_{0}^{1} d t^{\prime} \int_{c}^{t^{\prime}}[\cdot] d t^{\prime \prime}, \\
c \neq 1 \quad(c=0, \text { for simplicity }) .
\end{gathered}
$$


The first few terms of the stream function $f(t)$ are evaluated by implementing the previous algorithm and are listed in the following:

$$
\begin{aligned}
& f_{0}(t)= 0, \\
& f_{1}(t)=(1-\epsilon) t, \\
& f_{2}(t)=\left(\frac{\epsilon}{2}\right) t^{2}, \\
& f_{3}(t)=\left(\frac{1}{4}-\frac{\epsilon}{2}\right) t^{2}+\left(-\frac{1}{6}+\frac{2 \epsilon}{3}\right) t^{3}, \\
& f_{4}(t)=\left(\frac{\epsilon}{24}-\frac{\epsilon^{2}}{12}\right) t^{2}+\left(\frac{1}{4}-\frac{\epsilon}{2}\right) t^{3} \\
&+\left(-\frac{3}{16}+\frac{29 \epsilon}{28}+\frac{\epsilon^{2}}{24}\right) t^{4}, \\
& \quad+\left(\frac{7}{32}-\frac{41 \epsilon}{96}-\frac{\epsilon^{2}}{48}\right) t^{4} \\
& f_{5}(t)=t^{2}+\left(\frac{\epsilon}{24}-\frac{\epsilon^{2}}{12}\right) t^{3} \\
&+\left(-\frac{43}{240}+\frac{41 \epsilon}{40}+\frac{\epsilon^{2}}{15}\right) t^{5} .
\end{aligned}
$$

The desired $m$ th order approximate solution $\phi_{m}(\eta)$ obtained by Adomian's method is expressed as

$$
\phi_{m}(\eta)=\sum_{n=0}^{m-1} f_{n}(\eta)
$$

Hence, the approximate solutions $\phi_{3}(\eta), \phi_{5}(\eta)$, and $\phi_{7}(\eta)$ are, respectively, given in terms of the original variable $\eta$ as

$$
\begin{aligned}
\phi_{3}(\eta)= & (1-\epsilon)\left(1-e^{-\eta}\right)+\frac{1}{4}\left(1-e^{-\eta}\right)^{2} \\
& +\left(-\frac{1}{6}+\frac{2 \epsilon}{3}\right)\left(1-e^{-\eta}\right)^{3}, \\
\phi_{5}(\eta)= & (1-\epsilon)\left(1-e^{-\eta}\right)+\left(\frac{25}{96}+\frac{\epsilon}{48}-\frac{\epsilon^{2}}{12}\right)\left(1-e^{-\eta}\right)^{2} \\
& +\left(\frac{1}{12}+\frac{5 \epsilon}{24}-\frac{\epsilon^{2}}{12}\right)\left(1-e^{-\eta}\right)^{3} \\
& +\left(\frac{1}{32}+\frac{17 \epsilon}{96}+\frac{\epsilon^{2}}{48}\right)\left(1-e^{-\eta}\right)^{4} \\
& +\left(-\frac{43}{240}+\frac{41 \epsilon}{40}+\frac{\epsilon^{2}}{15}\right)\left(1-e^{-\eta}\right)^{5},
\end{aligned}
$$

$$
\begin{aligned}
\phi_{7}(\eta)= & (1-\epsilon)\left(1-e^{-\eta}\right) \\
& +\left(\frac{171}{640}+\frac{\epsilon}{72}-\frac{553 \epsilon^{2}}{5760}-\frac{\epsilon^{3}}{960}\right)\left(1-e^{-\eta}\right)^{2} \\
& +\left(\frac{47}{480}+\frac{11 \epsilon}{60}-\frac{53 \epsilon^{2}}{576}+\frac{\epsilon^{3}}{1440}\right)\left(1-e^{-\eta}\right)^{3} \\
& +\left(\frac{31}{768}+\frac{451 \epsilon}{2304}-\frac{59 \epsilon^{2}}{1152}-\frac{\epsilon^{3}}{288}\right)\left(1-e^{-\eta}\right)^{4} \\
& +\left(\frac{11}{960}+\frac{137 \epsilon}{720}-\frac{7 \epsilon^{2}}{288}-\frac{\epsilon^{3}}{240}\right)\left(1-e^{-\eta}\right)^{5} \\
& +\left(\frac{31}{5760}+\frac{367 \epsilon}{2880}+\frac{65 \epsilon^{2}}{1152}+\frac{\epsilon^{3}}{960}\right)\left(1-e^{-\eta}\right)^{6} \\
& +\left(-\frac{13}{8960}+\frac{5921 \epsilon}{40320}-\frac{83 \epsilon^{2}}{24192}+\frac{89 \epsilon^{3}}{34560}-\frac{19 \epsilon^{4}}{120960}\right) \\
& \times\left(1-e^{-\eta}\right)^{7} .
\end{aligned}
$$

Here, we refer to that the series solution obtained previous leads to an exact solution at $\epsilon=0.5$. In this case, the approximate solutions become

$$
\begin{aligned}
\phi_{3}(\eta)= & \frac{1}{2}\left(1-e^{-\eta}\right)+\frac{1}{4}\left(1-e^{-\eta}\right)^{2}+\frac{1}{6}\left(1-e^{-\eta}\right)^{3} \\
= & \frac{1}{2} \sum_{r=1}^{3} \frac{1}{r}\left(1-e^{-\eta}\right)^{r}, \\
\phi_{5}(\eta)= & \frac{1}{2}\left(1-e^{-\eta}\right)+\frac{1}{4}\left(1-e^{-\eta}\right)^{2}+\frac{1}{6}\left(1-e^{-\eta}\right)^{3} \\
& +\frac{1}{8}\left(1-e^{-\eta}\right)^{4}+\frac{1}{10}\left(1-e^{-\eta}\right)^{5} \\
= & \frac{1}{2} \sum_{r=1}^{5} \frac{1}{r}\left(1-e^{-\eta}\right)^{r}, \\
\phi_{7}(\eta)= & \frac{1}{2}\left(1-e^{-\eta}\right)+\frac{1}{4}\left(1-e^{-\eta}\right)^{2}+\frac{1}{6}\left(1-e^{-\eta}\right)^{3} \\
& +\frac{1}{8}\left(1-e^{-\eta}\right)^{4}+\frac{1}{10}\left(1-e^{-\eta}\right)^{5} \\
& +\frac{1}{12}\left(1-e^{-\eta}\right)^{6}+\frac{1}{14}\left(1-e^{-\eta}\right)^{7} \\
= & \frac{1}{2} \sum_{r=1}^{7} \frac{1}{r}\left(1-e^{-\eta}\right)^{r} .
\end{aligned}
$$

Therefore, the $m$-term series solution is given by

$$
\phi_{m}(\eta)=\frac{1}{2} \sum_{r=1}^{m} \frac{1}{r}\left(1-e^{-\eta}\right)^{r},
$$


and thus, the following exact solution is obtained as $m \rightarrow \infty$ :

$$
\begin{aligned}
f(\eta) & =\lim _{m \rightarrow \infty} \phi_{m}(\eta) \\
& =\frac{1}{2} \sum_{r=1}^{\infty} \frac{1}{r}\left(1-e^{-\eta}\right)^{r} \\
& =-\frac{1}{2} \ln \left[1-\left(1-e^{-\eta}\right)\right]=\frac{\eta}{2} .
\end{aligned}
$$

This exact solution satisfies the boundary conditions and can be easily verified by direct substitution. For more validation, the results obtained by the present technique are checked here via a comparison with those published in the literature. It is well known that at $\epsilon=1$, the problem reduces to one of the two forms of the Blasius problem. In that case, the skinfriction coefficient is computed by many authors as discussed in Section 5.

4.2. Special Class of the Falkner-Skan Problem. Here, the proposed approach is applied to a special class of the FalknerSkan problem. As mentioned before, this special class is given by (4)-(5) at $\gamma=1$. Proceeding as in the previous example, the approximate solution can be obtained by using the recurrence scheme:

$$
\begin{gathered}
u_{0}(t)=1-\epsilon, \\
f_{0}(t)=0 \\
u_{1}(t)=(2 \epsilon-1) t \\
f_{1}(t)=(1-\epsilon) t \\
u_{n+1}(t)=L^{-1}\left[\sum_{i=0}^{n} t^{n-i} u_{i}^{\prime}(t)-\sum_{j=0}^{n} \sum_{i=0}^{j} t^{n-j} f_{i}(t) u_{j-i}^{\prime}(t)\right], \\
f_{n+1}(t)=\int_{0}^{t}\left(\frac{1}{1-z}\right) u_{n}(z) d z, \quad n \geq 1, \quad c \neq 1 . \\
L^{-1}[\cdot]=\int_{0}^{t} d t^{\prime} \int_{c}^{t^{\prime}}[\cdot] d t^{\prime \prime}-t \int_{0}^{1} d t^{\prime} \int_{c}^{t^{\prime}}[\cdot] d t^{\prime \prime}, \quad c \neq 1
\end{gathered}
$$

The first few terms of the stream function $f(t)$ are evaluated by implementing the algorithm given in (32) and are listed in the following:

$$
\begin{aligned}
f_{0}(t)=0 \\
f_{1}(t)=(1-\epsilon) t \\
f_{2}(t)=\left(\frac{\epsilon}{2}\right) t^{2}, \\
f_{3}(t)=\left(\frac{1}{4}-\frac{\epsilon}{2}\right) t^{2}+\left(-\frac{1}{6}+\frac{2 \epsilon}{3}\right) t^{3}, \\
f_{4}(t)=\left(-\frac{1}{24}+\frac{\epsilon}{6}-\frac{\epsilon^{2}}{6}\right) t^{2}+\left(\frac{1}{4}-\frac{\epsilon}{2}\right) t^{3} \\
\quad+\left(-\frac{1}{6}+\frac{13 \epsilon}{24}+\frac{\epsilon^{2}}{12}\right) t^{4},
\end{aligned}
$$

$$
\begin{aligned}
f_{5}(t)=( & \left.-\frac{1}{24}+\frac{\epsilon}{6}-\frac{\epsilon^{2}}{6}\right) t^{3}+\left(\frac{5}{24}-\frac{19 \epsilon}{48}-\frac{\epsilon^{2}}{24}\right) t^{4} \\
& +\left(-\frac{17}{120}+\frac{5 \epsilon}{12}+\frac{2 \epsilon^{2}}{15}\right) t^{5}
\end{aligned}
$$

Hence, the approximate solutions $\phi_{3}(\eta), \phi_{5}(\eta)$, and $\phi_{7}(\eta)$ are, respectively, given in terms of the original variable $\eta$ as

$$
\begin{aligned}
\phi_{3}(\eta)= & (1-\epsilon)\left(1-e^{-\eta}\right)+\frac{1}{4}\left(1-e^{-\eta}\right)^{2} \\
& +\left(-\frac{1}{6}+\frac{2 \epsilon}{3}\right)\left(1-e^{-\eta}\right)^{3}, \\
\phi_{5}(\eta)= & (1-\epsilon)\left(1-e^{-\eta}\right)+\left(\frac{5}{24}+\frac{\epsilon}{6}-\frac{\epsilon^{2}}{6}\right)\left(1-e^{-\eta}\right)^{2} \\
& +\left(\frac{1}{24}+\frac{\epsilon}{3}-\frac{\epsilon^{2}}{6}\right)\left(1-e^{-\eta}\right)^{3} \\
& +\left(\frac{1}{24}+\frac{7 \epsilon}{48}+\frac{\epsilon^{2}}{24}\right)\left(1-e^{-\eta}\right)^{4} \\
& +\left(-\frac{17}{120}+\frac{5 \epsilon}{12}+\frac{2 \epsilon^{2}}{15}\right)\left(1-e^{-\eta}\right)^{5}, \\
\phi_{7}(\eta)= & (1-\epsilon)\left(1-e^{-\eta}\right) \\
& +\left(-\frac{67}{630}+\frac{25 \epsilon}{84}+\frac{181 \epsilon^{2}}{1680}+\frac{41 \epsilon^{3}}{2520}\right)\left(1-e^{-\eta}\right)^{7} . \\
& +\left(\frac{191}{960}+\frac{19 \epsilon}{96}-\frac{91 \epsilon^{2}}{480}-\frac{\epsilon^{3}}{240}\right)\left(1-e^{-\eta}\right)^{2} \\
& +\left(\frac{13}{360}+\frac{17 \epsilon}{48}-\frac{3 \epsilon^{2}}{16}+\frac{\epsilon^{3}}{360}\right)\left(1-e^{-\eta}\right)^{3} \\
& \left.+\frac{\epsilon^{2}}{40}-\frac{\epsilon^{3}}{60}\right)\left(1-e^{-\eta}\right)^{5} \\
& \left.+\frac{\epsilon^{3}}{72}\right)\left(1-e^{-\eta}\right)^{4} \\
& \left(\frac{13 \epsilon}{288}+\frac{31 \epsilon^{2}}{288}+\frac{\epsilon^{3}}{240}\right)\left(1-e^{-\eta}\right)^{6} \\
& \left(\frac{17}{30}\right)(134)
\end{aligned}
$$

The effectiveness of the present technique is used here not only to obtain the exact solution of the Falkner-Skan equation at $\delta=0$ and $\epsilon=0.5$ but also to get numerical solutions with good accuracy. On inserting $\epsilon=0.5$ into the approximate solutions given by (34), we have

$$
\begin{aligned}
\phi_{3}(\eta) & =\frac{1}{2}\left(1-e^{-\eta}\right)+\frac{1}{4}\left(1-e^{-\eta}\right)^{2}+\frac{1}{6}\left(1-e^{-\eta}\right)^{3} \\
& =\frac{1}{2} \sum_{r=1}^{3} \frac{1}{r}\left(1-e^{-\eta}\right)^{r},
\end{aligned}
$$




$$
\begin{aligned}
\phi_{5}(\eta)= & \frac{1}{2}\left(1-e^{-\eta}\right)+\frac{1}{4}\left(1-e^{-\eta}\right)^{2}+\frac{1}{6}\left(1-e^{-\eta}\right)^{3} \\
& +\frac{1}{8}\left(1-e^{-\eta}\right)^{4}+\frac{1}{10}\left(1-e^{-\eta}\right)^{5} \\
= & \frac{1}{2} \sum_{r=1}^{5} \frac{1}{r}\left(1-e^{-\eta}\right)^{r}, \\
\phi_{7}(\eta)= & \frac{1}{2}\left(1-e^{-\eta}\right)+\frac{1}{4}\left(1-e^{-\eta}\right)^{2}+\frac{1}{6}\left(1-e^{-\eta}\right)^{3} \\
& +\frac{1}{8}\left(1-e^{-\eta}\right)^{4}+\frac{1}{10}\left(1-e^{-\eta}\right)^{5} \\
& +\frac{1}{12}\left(1-e^{-\eta}\right)^{6}+\frac{1}{14}\left(1-e^{-\eta}\right)^{7} \\
= & \frac{1}{2} \sum_{r=1}^{7} \frac{1}{r}\left(1-e^{-\eta}\right)^{r} .
\end{aligned}
$$

As indicated in Section 4.1, these approximate solutions lead to the same exact solution given by $(31): f(\eta)=\eta / 2$ in the limit.

\section{Results and Discussion}

At $\epsilon=1$, Bairstow [29] found that $f^{\prime \prime}(0)=0.335$ using a power series, whereas Goldstein [30] obtained $f^{\prime \prime}(0)=$ 0.332. Besides, using a finite difference method, Falkner [31] computed that $f^{\prime \prime}(0)=0.3325765$, and Horwarth [32] yields that $f^{\prime \prime}(0)=0.332057$. In [33], Fazio computed that $f^{\prime \prime}(0)=0.332057336215$. Also, in [34] Boyd used Töpfer's algorithm to obtain the accurate value $f^{\prime \prime}(0)=$ 0.33205733621519630 . Adomain's method was implemented in [35] by Abbasbandy, and it was found that $f^{\prime \prime}(0)=$ 0.333329 , whereas a variational iteration method with the Padé approximants allows Wazwaz [6] to calculate the value $f^{\prime \prime}(0)=0.3732905625$. Tajvidi et al. [28] apply the modified rational Legendre functions to get a value of $f^{\prime \prime}(0)=0.33209$. The values of the skin-friction coefficient are given in Table 1 at $\epsilon=0,0.1,0.2,0.3,0.4,0.5,0.6,0.7,0.8,0.9$, and 1 using 11,13 , and 15 terms of the series (27). The current method finds that the skin-friction at $\epsilon=1$ approximately equals $\phi_{15}^{\prime \prime}(0)=0.331775$, which is very close to those values discussed previously.

Regarding the stream function $f(\eta)$, it is plotted in Figure 1 using 15 terms, and the fluid velocity $f^{\prime}(\eta)$ is depicted in Figure 2 using the approximate solutions $\phi_{7}(\eta), \phi_{9}(\eta)$, $\phi_{11}(\eta), \phi_{13}(\eta)$, and $\phi_{15}(\eta)$ at $\epsilon=0,0.1,0.2,0.3,0.4,0.5,0.6$, $0.7,0.8,0.9$, and 1 . It is observed from Figure 2 that the approximate solutions using a few terms of Adomian's series converge rapidly to a certain curve at some values of the parameter $\epsilon$.

The exact solution $f(\eta)=\eta / 2$ obtained in Section 4.2 for the Falkner-Skan equation at $\epsilon=0.5$ has been reported very recently by Kudenatti [27]. He has derived the exact solution to the Falkner-Skan equation for general values of the pressure gradient parameter $\delta$. In order to check the accuracy of our approach, the values of the skin-friction coefficient are
TABLE 1: The approximate values of the skin-friction coefficient $f^{\prime \prime}(0)$ for the class of the Blasius problem using 11,13 , and 15 terms of Adomian's series.

\begin{tabular}{lccc}
\hline$\epsilon$ & $\phi_{11}^{\prime \prime}(0)$ & $\phi_{13}^{\prime \prime}(0)$ & $\phi_{15}^{\prime \prime}(0)$ \\
\hline 0.0 & -0.456523 & -0.454506 & -0.453122 \\
0.1 & -0.356731 & -0.354995 & -0.353838 \\
0.2 & -0.261082 & -0.259650 & -0.258715 \\
0.3 & -0.169677 & -0.168611 & -0.167920 \\
0.4 & -0.082616 & -0.082014 & -0.081624 \\
0.5 & 0.000000 & 0.000000 & 0.000000 \\
0.6 & 0.078072 & 0.077292 & 0.076775 \\
0.7 & 0.151503 & 0.149723 & 0.148522 \\
0.8 & 0.220194 & 0.217151 & 0.215060 \\
0.9 & 0.284048 & 0.279437 & 0.276206 \\
1.0 & 0.342969 & 0.336441 & 0.331775 \\
\hline
\end{tabular}

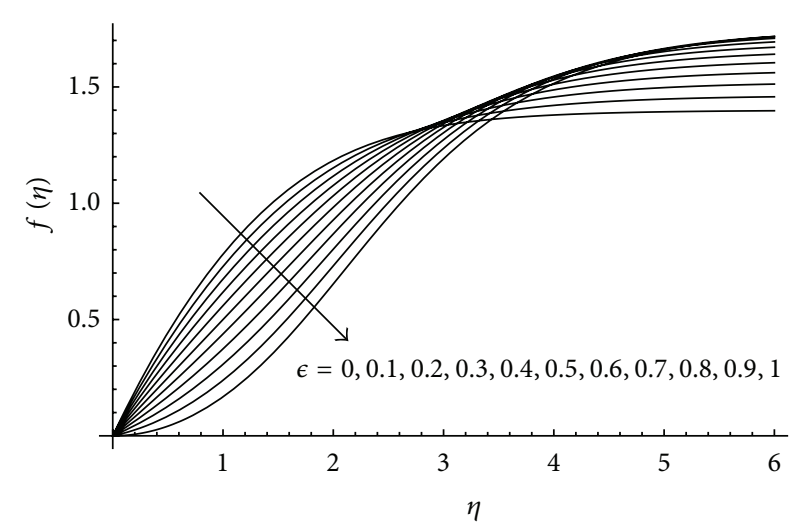

FIgURE 1: The stream function for the class of the Blasius problem at different values of $\epsilon$ using 15 terms of the current method.

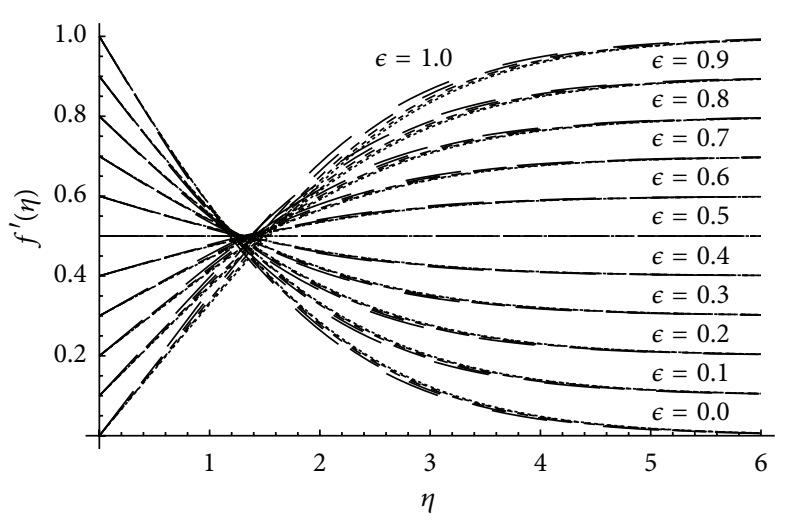

Figure 2: The fluid velocity for the class of the Blasius problem at different values of $\epsilon$ using 7, 9, 11, 13, and 15 terms of the current method.

compared in Table 2 with those exactly obtained by Kudenatti [27] in the range $0<\epsilon<0.5$. The results reveal that a good agreement has been achieved via the present approach. In addition, the stream function $f(\eta)$ is graphed in Figure 3 at several values of the parameter $\epsilon$ by using 15 terms of the decomposition series. At the same values and in Figure 4, 
TABLE 2: The approximate values of the skin-friction coefficient $f^{\prime \prime}(0)$ for the class of the Falkner-Skan problem using 11, 15, and 33 terms of Adomian's series.

\begin{tabular}{ccccc}
\hline$\epsilon$ & $\phi_{11}^{\prime \prime}(0)$ & $\phi_{15}^{\prime \prime}(0)$ & $\phi_{33}^{\prime \prime}(0)$ & $\begin{array}{c}\text { Exact values } \\
\text { Reference [28] }\end{array}$ \\
\hline 0.0 & -0.617661 & -0.622494 & -0.625945 & -0.627504 \\
0.1 & -0.479542 & -0.485036 & -0.490729 & -0.492625 \\
0.2 & -0.348276 & -0.353437 & -0.360126 & -0.363901 \\
0.3 & -0.224304 & -0.228296 & -0.234485 & -0.237219 \\
0.4 & -0.108067 & -0.110256 & -0.114249 & -0.115811 \\
0.5 & 0.000000 & 0.000000 & 0.000000 & 0.000000 \\
0.6 & 0.099467 & 0.101753 & 0.107483 & \\
0.7 & 0.189909 & 0.194253 & 0.207152 & \\
0.8 & 0.270907 & 0.276719 & 0.297613 & \\
0.9 & 0.342046 & 0.34835 & 0.377038 & \\
1.0 & 0.402921 & 0.408321 & 0.443088 & \\
\hline
\end{tabular}

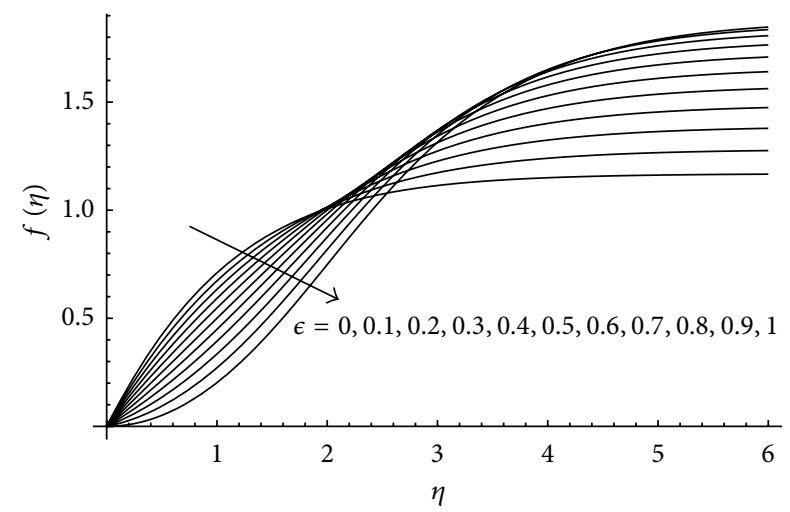

Figure 3: The stream function for the class of the Falkner-Skan problem at different values of $\epsilon$ using 15 terms of the current method.

the fluid velocity is depicted by using the approximate solutions $\phi_{11}(\eta), \phi_{13}(\eta)$, and $\phi_{15}(\eta)$. It can be concluded from Figure 4 that our results are a coincidence with those exactly obtained in [27] at the values $\epsilon=0.1,0.2,0.3,0.4,0.5,0.7$, and 1 , while the fluid velocity at the other values $\epsilon=0,0.6,0.8$, and 0.9 was not discussed by Kudenatti [27].

\section{Conclusion}

An approach is presented in this paper to treat the boundary condition at infinity which is the main feature of the boundary layer equations. The suggested approach is based on changing the boundary condition at infinity to a classical one by the help of a transformation. The current approach is applied to solve a class of the Blasius problem and a special class of the Falkner-Skan problem via an improved version of Adomian's method. Moreover, exact solutions are deduced at a certain value of the velocity ratio parameter $\epsilon$. In addition, the current numerical results are compared with the other published solutions, where good agreement is found. One of

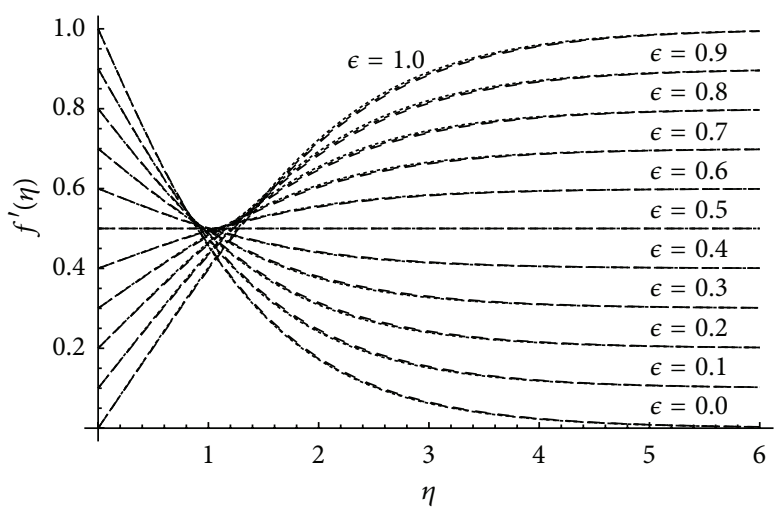

FIGURE 4: The fluid velocity for the class of the Falkner-Skan problem at different values of $\epsilon$ using 11,13, and 15 terms of the current method.

the main advantages of the present approach is the avoidance of the Padé approximation to deal with the infinity boundary condition.

\section{References}

[1] Z. Belhachmi, B. Brighi, and K. Taous, "On the concave solutions of the Blasius equation," Acta Mathematica Universitatis Comenianae, vol. 69, no. 2, pp. 199-214, 2000.

[2] B. K. Datta, "Analytic solution for the Blasius equation," Indian Journal of Pure and Applied Mathematics, vol. 34, no. 2, pp. 237240, 2003.

[3] H. K. Kuiken, "On boundary layers in fluid mechanics that decay algebraically along stretches of wall that are not vanishingly small," IMA Journal of Applied Mathematics, vol. 27, no. 4, pp. 387-405, 1981.

[4] H. K. Kuiken, "A "backward" free-convective boundary layer," The Quarterly Journal of Mechanics and Applied Mathematics, vol. 34, no. 3, pp. 397-413, 1981.

[5] J. He, "Approximate analytical solution of Blasius' equation," Communications in Nonlinear Science and Numerical Simulation, vol. 3, no. 4, pp. 260-263, 1998.

[6] A.-M. Wazwaz, "The variational iteration method for solving two forms of Blasius equation on a half-infinite domain," Applied Mathematics and Computation, vol. 188, no. 1, pp. 485491, 2007.

[7] B. I. Yun, "Intuitive approach to the approximate analytical solution for the Blasius problem," Applied Mathematics and Computation, vol. 215, no. 10, pp. 3489-3494, 2010.

[8] S. Abbasbandy and C. Bervillier, "Analytic continuation of Taylor series and the boundary value problems of some nonlinear ordinary differential equations," Applied Mathematics and Computation, vol. 218, no. 5, pp. 2178-2199, 2011.

[9] M. Khan and M. A. Gondal, "Homotopy perturbation Padé transform method for Blasius flow equation using He's polynomials," International Journal of Nonlinear Sciences and Numerical Simulation, vol. 12, no. 1-8, pp. 1-7, 2011.

[10] M. Khan, M. A. Gondal, I. Hussain, and S. K. Vanani, "A new comparative study between homotopy analysis transform method and homotopy perturbation transform method on a semi infinite domain," Mathematical and Computer Modelling, vol. 55, no. 3-4, pp. 1143-1150, 2012. 
[11] R. Fazio, "Blasius problem and Falkner-Skan model: Töpfer's algorithm and its extension," Computers and Fluids, vol. 73, pp. 202-209, 2013.

[12] G. Adomian, Solving Frontier Problems of Physics: The Decomposition Method, Kluwer Academic Publishers, Boston, Mass, USA, 1994.

[13] A.-M. Wazwaz, R. Rach, and J.-S. Duan, "Adomian decomposition method for solving the Volterra integral form of the LaneEmden equations with initial values and boundary conditions," Applied Mathematics and Computation, vol. 219, no. 10, pp. 5004-5019, 2013.

[14] A. M. Wazwaz, R. Rach, and J. S. Duan, "A study on the systems of the Volterra integral forms of the Lane-Emden equations by the Adomian decomposition method," Mathematical Methods in the Applied Sciences, 2013.

[15] A. E.-H. Ebaid, "Approximate periodic solutions for the nonlinear relativistic harmonic oscillator via differential transformation method," Communications in Nonlinear Science and Numerical Simulation, vol. 15, no. 7, pp. 1921-1927, 2010.

[16] A. E. Ebaid, "A reliable aftertreatment for improving the differential transformation method and its application to nonlinear oscillators with fractional nonlinearities," Communications in Nonlinear Science and Numerical Simulation, vol. 16, no. 1, pp. 528-536, 2011.

[17] S. N. Venkatarangan and K. Rajalakshmi, "Modification of Adomian's decomposition method to solve equations containing radicals," Computers \& Mathematics with Applications, vol. 29, no. 6, pp. 75-80, 1995.

[18] A.-M. Wazwaz, "The modified decomposition method and Padé approximants for solving the Thomas-Fermi equation," Applied Mathematics and Computation, vol. 105, no. 1, pp. 11-19, 1999.

[19] A.-M. Wazwaz, "The modified decomposition method and Padé approximants for a boundary layer equation in unbounded domain," Applied Mathematics and Computation, vol. 177, no. 2, pp. 737-744, 2006.

[20] S. A. Kechil and I. Hashim, "Non-perturbative solution of freeconvective boundary-layer equation by Adomian decomposition method," Physics Letters A, vol. 363, no. 1-2, pp. 110-114, 2007.

[21] S. Awang Kechil and I. Hashim, "Series solution of flow over nonlinearly stretching sheet with chemical reaction and magnetic field," Physics Letters A, vol. 372, no. 13, pp. 2258-2263, 2008.

[22] E. Alizadeh, K. Sedighi, M. Farhadi, and H. R. Ebrahimi-Kebria, "Analytical approximate solution of the cooling problem by Adomian decomposition method," Communications in Nonlinear Science and Numerical Simulation, vol. 14, no. 2, pp. 462-472, 2009.

[23] T. Hayat, Q. Hussain, and T. Javed, "The modified decomposition method and Padé approximants for the MHD flow over a non-linear stretching sheet," Nonlinear Analysis. Real World Applications, vol. 10, no. 2, pp. 966-973, 2009.

[24] M. M. Rashidi and S. A. Mohimanian Pour, "Explicit solution of axisymmetric stagnation flow towards a shrinking sheet by DTM-Padé," Applied Mathematical Sciences, vol. 4, no. 53-56, pp. 2617-2632, 2010.

[25] M. M. Rashidi and M. Keimanesh, "Using differential transform method and padé approximant for solving mhd flow in a laminar liquid film from a horizontal stretching surface," Mathematical Problems in Engineering, vol. 2010, Article ID 491319, 14 pages, 2010.
[26] A. Ebaid, "A new analytical and numerical treatment for singular two-point boundary value problems via the Adomian decomposition method," Journal of Computational and Applied Mathematics, vol. 235, no. 8, pp. 1914-1924, 2011.

[27] R. B. Kudenatti, "A new exact solution for boundary layer flow over a stretching plate," International Journal of Non-Linear Mechanics, vol. 47, pp. 727-733, 2012.

[28] T. Tajvidi, M. Razzaghi, and M. Dehghan, "Modified rational Legendre approach to laminar viscous flow over a semi-infinite flat plate," Chaos, Solitons and Fractals, vol. 35, no. 1, pp. 59-66, 2008.

[29] L. Bairstow, "Skin friction," Journal of the Royal Aeronautical Society, vol. 29, pp. 3-23, 1925.

[30] S. Goldstein, "Concerning some solutions of the boundary layer equations in hydrodynamics," Proceedings of the Cambridge Philosophical Society, vol. 26, pp. 1-30, 1930.

[31] V. M. Falkner, "A method of numerical solution of differential equations," Philosophical Magazine, vol. 21, pp. 624-640, 1936.

[32] L. Horwarth, "On the solution of the laminar boundary layer equations," Proceedings of the Royal Society of London A, vol. 164, pp. 547-579, 1938.

[33] R. Fazio, "The Blasius problem formulated as a free boundary value problem," Acta Mechanica, vol. 95, no. 1-4, pp. 1-7, 1992.

[34] J. P. Boyd, "The Blasius function in the complex plane," Experimental Mathematics, vol. 8, no. 4, pp. 381-394, 1999.

[35] S. Abbasbandy, "A numerical solution of Blasius equation by Adomian's decomposition method and comparison with homotopy perturbation method," Chaos, Solitons and Fractals, vol. 31, no. 1, pp. 257-260, 2007. 


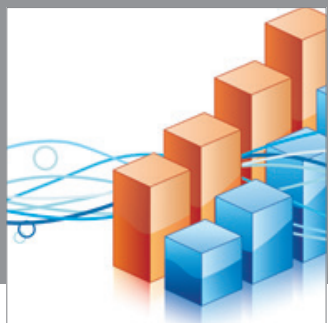

Advances in

Operations Research

mansans

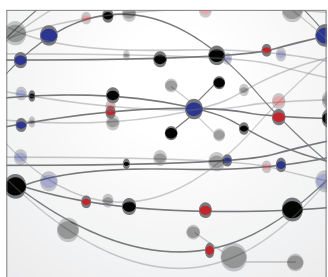

The Scientific World Journal
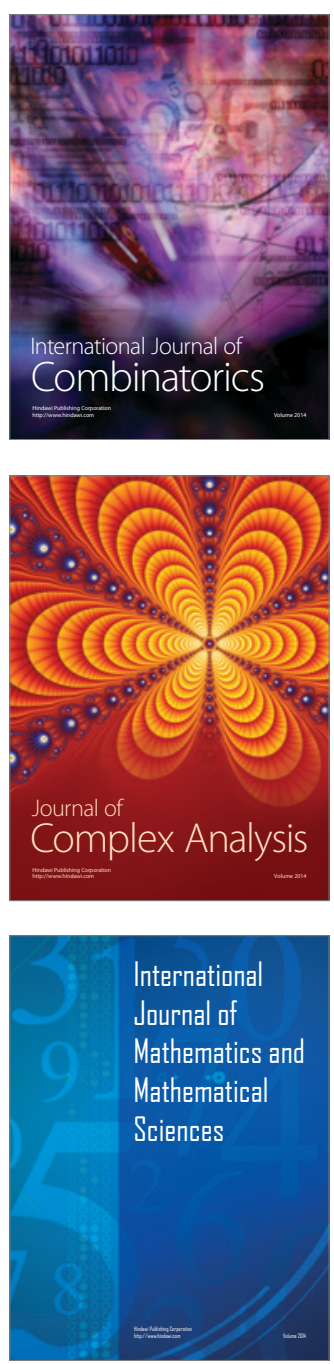
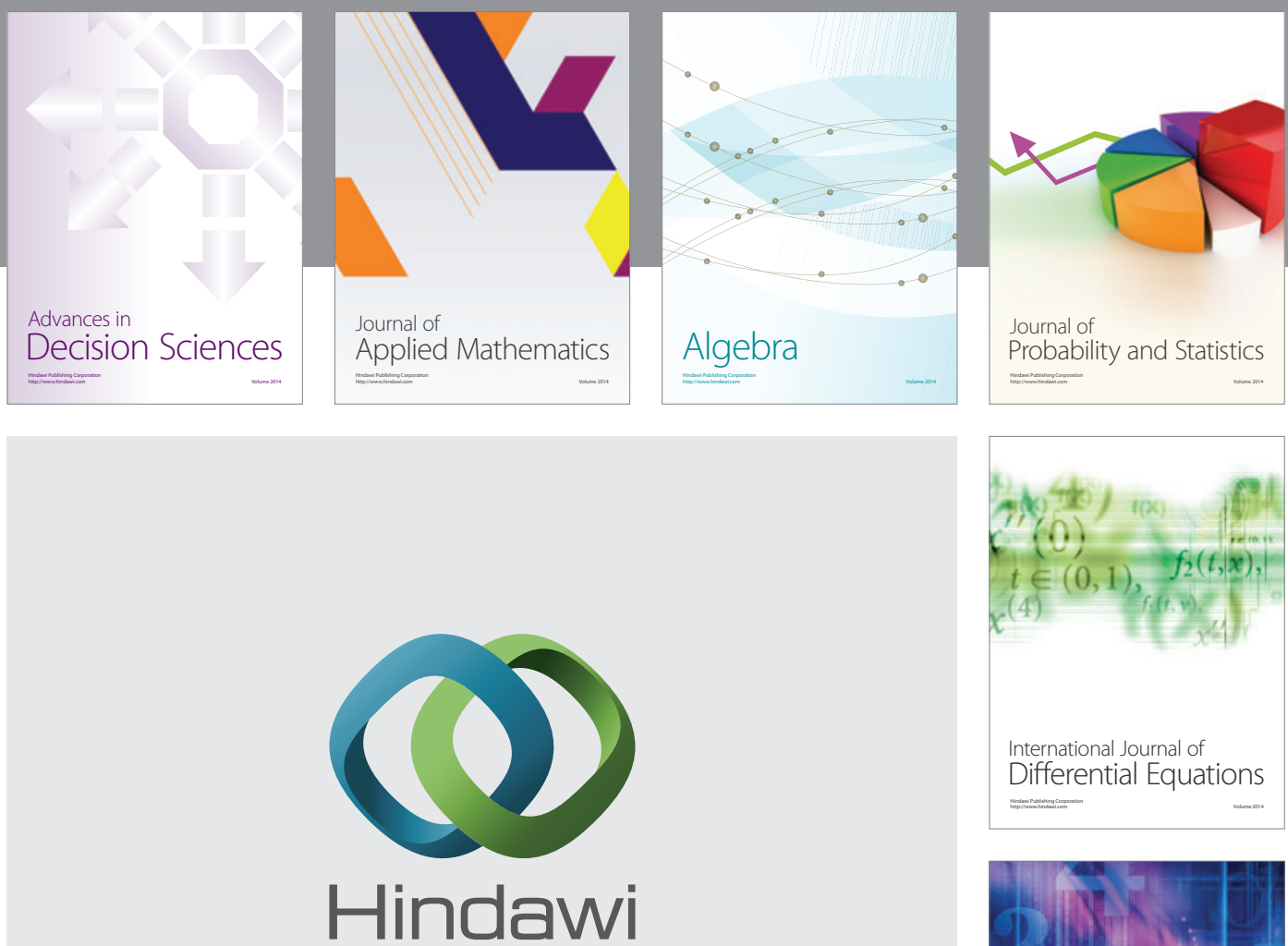

Submit your manuscripts at http://www.hindawi.com
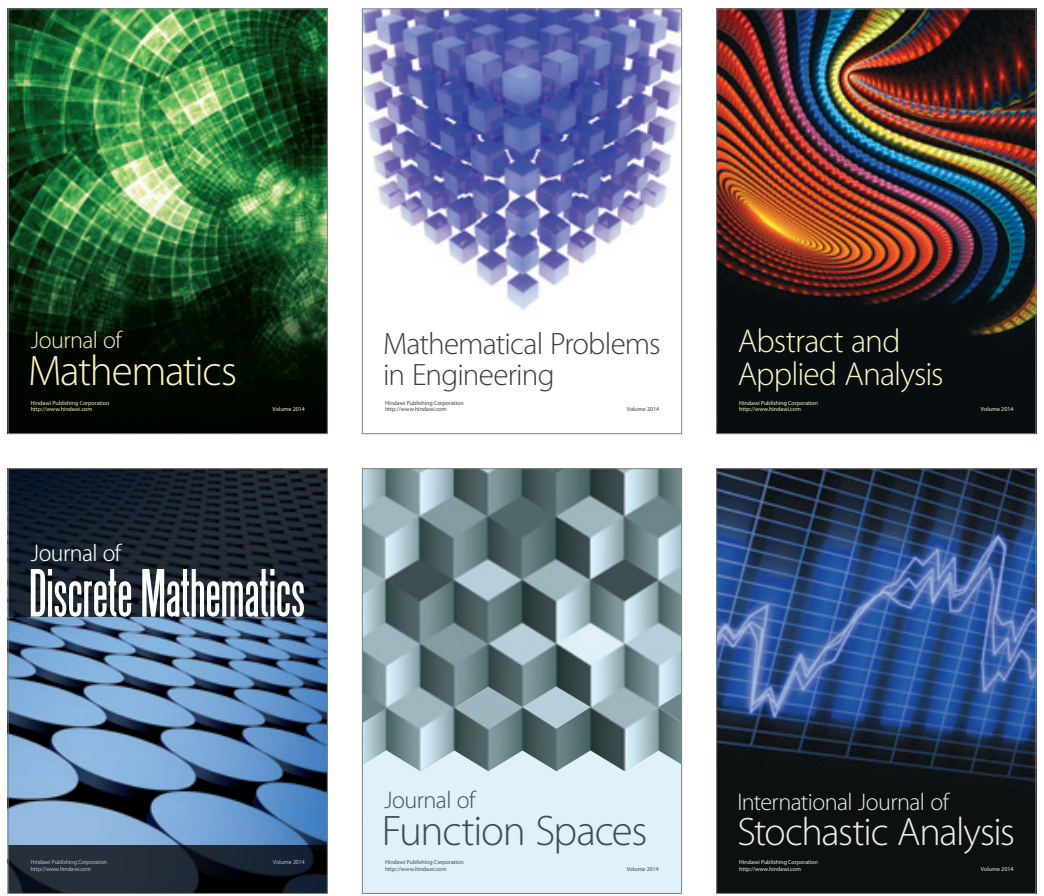

Journal of

Function Spaces

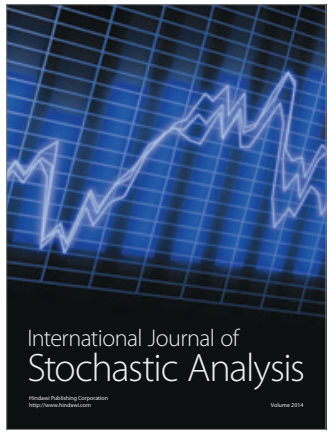

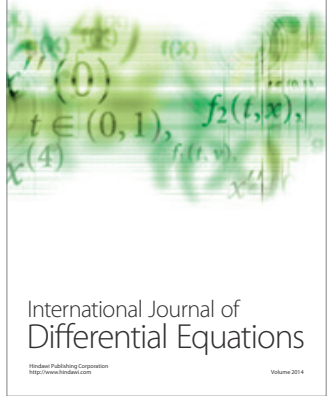
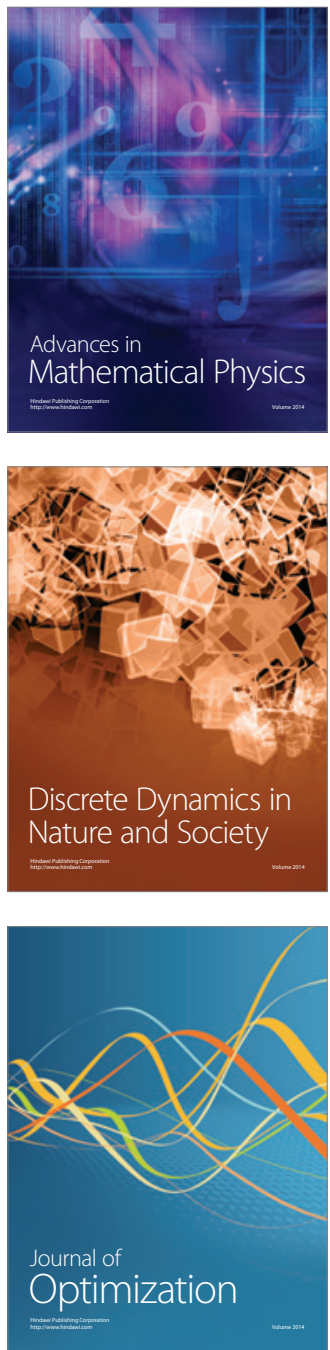